\title{
Downregulating microRNA-144 mediates a metabolic shift in lung cancer cells by regulating GLUT1 expression
}

\author{
MIN LIU, JUN GAO, QIN HUANG, YANFENG JIN and ZHONGHUA WEI \\ Department of Pathology, Shanghai Jiao Tong University Affiliated Sixth People's Hospital, Shanghai 200233, P.R. China
}

Received January 6, 2015; Accepted February 1, 2016

DOI: $10.3892 / \mathrm{ol} .2016 .4468$

\begin{abstract}
Lung cancer is the leading cause of cancer-associated mortality worldwide. Increasing evidence has found that cancer metabolism alternations represent a critical hallmark for lung cancer. There is an urgent requirement to understand and dissect the molecular mechanisms underlying cancer metabolism for lung cancer therapy. It remains largely unknown whether the deregulation of miRNAs contributes to the cancer metabolism. The present study aimed to investigate the role of miR-144 in lung cancer. Glucose uptake rate and lactate production assays demonstrated that miR-144 expression is decreased and therefore enhances the aerobic metabolism in lung cancer cells. In addition, western blot analysis revealed that miR-144 performs this function by increasing the expression of glucose transporter 1 (GLUT1), leading to an increase in glucose uptake and lactate production. Furthermore, cell viability assays demonstrated that the altered metabolism induced by miR-144 results in the rapid growth of cancer cells. In conclusion, these results identify miR-144 as a molecular switch involved in the orchestration of the Warburg effect in lung cancer cells via targeting the expression of GLUT1.
\end{abstract}

\section{Introduction}

Lung cancer is the main cause of cancer-related mortality worldwide, and is pathologically classified as one of two types, namely, small cell (15\%) or non-small cell (85\%) lung cancer (1). The disease is notoriously fatal at an advanced stage, with a 5-year survival rate of $<15 \%(1,2)$. Therefore, an improved understanding of the molecular mechanisms involved in the development of lung cancer is urgently required as the basis of the identification of novel therapeutic targets and the development of novel treatment strategies.

The metabolism of cancer cells is significantly different from that of normally differentiated cells (3). Normally

Correspondence to: Dr Zhonghua Wei, Department of Pathology, Shanghai Jiao Tong University Affiliated Sixth People's Hospital, 600 Yishan Road, Shanghai 200233, P.R. China

E-mail: weizhonghua365@163.com

Key words: microRNA-144, GLUT1, glycolysis, lung cancer differentiated cells rely primarily on the oxidation of pyruvate in the mitochondria to generate energy for cellular physiological functions; however, even with sufficient oxygen, rapidly growing cancer cells rely on aerobic glycolysis to generate energy (4). This phenomenon is termed 'the Warburg effect' (5). This metabolic shift towards enhanced glycolysis is hypothesized to be the result of adaptations to support the continuous proliferation of cancer cells. The upregulation of specific glucose transporter 1 (GLUT1) may represent a key mechanism by which malignant cells may achieve increased glucose uptake to support the high rate of glycolysis. There is a growing body of evidence indicating that reprogrammed cancer metabolism is a potential therapeutic target (5).

microRNAs (miRs/miRNAs) are an evolutionarily conserved group of small (18-24-nucleotide) non-coding RNAs, which suppress gene expression in a sequence-specific manner (6). miRNAs negatively regulate the expression of target genes through complementarity between the miRNA seed sequence and the 3'-untranslated region (UTR) of the target mRNA (6). Target mRNA is degraded when miRNAs bind with exact complementarity to the protein-encoding messenger RNA (mRNA), while mRNA translation is repressed when miRNAs exhibit inexact complementarity to the 3'UTR of the target mRNA (7).

miRNAs are now known as essential regulators of development and physiological processes (7). By regulating oncogenic and tumor suppressor signal pathways, miRNAs may also play significant roles in cancer development. Recently, a large number of miRNAs were identified as important natural regulators of metabolism (8). For example, miR-33a and miR-33b play a key role in regulating fatty acid degradation and cholesterol homeostasis (9), while numerous miRNAs, such as miR-21, miR-143, miR-130 and miR-27a, are reported to be involved in white-adipocyte differentiation. Furthermore, the inhibition of miR-122 is believed to decrease plasma triglyceride and cholesterol concentrations (10).

An increasing level of attention has been conferred on the role of microRNA-144 in tumorigenesis and the treatment of cancer. A number of studies have reported miR-144 downregulation in a range of cancer types, including osteosarcoma and mesothelioma, indicating that the miRNA is a potential tumor suppressor $(11,12)$. In particular, one study revealed an inverse correlation between the level of miR-144 and the development of gastric cancer (13), while another study reported an elevated miR-144 level in colorectal cancer (14). A study by 
$\mathrm{Fu}$ et al showed that cell proliferation, migration and invasion is promoted by miR-144 in nasopharyngeal carcinoma by the suppression of PTEN expression (15). Consequently, miR-144 appears to have a complex and highly tissue-specific function in tumorigenesis and cancer development.

The direct role of miR-144 in lung cancer cells has not yet been reported. In the present study, it was found that miR-144 is significantly decreased in lung cancer. The loss of miR-144 function enhanced the proliferation of the tumor cells via increased glycolysis through the direct targeting of the 3'-UTR of GLUT1.

\section{Materials and methods}

Cell culture. A549 and HEK293T cells were purchased from the American Type Culture Collection and cultured in Dulbecco' modified Eagle's medium (DMEM) supplemented with $10 \%$ fetal bovine serum (FBS; Invitrogen; Thermo Fisher Scientific, Inc., Waltham, MA, USA), $4.5 \mathrm{~g} / 1$ glucose and $100 \mathrm{U} / \mathrm{ml}$ penicillin/streptomycin in a tissue incubator maintained at $37^{\circ} \mathrm{C}$.

Transfections. All transfections were performed using Lipofectamine 2000 (Invitrogen; Thermo Fisher Scientific, Inc.) according to the manufacturer's protocols. The miR-144 mimics or inhibitor and the negative controls were purchased from GenePharma (Shanghai, China). The miR-144 mimics and miR-144 inhibitor were used at a final concentration of $50 \mathrm{nM}$

Luciferase reporter assay. For the luciferase reporter assay, HEK293T cells were seeded in a 24-well plate and were grown to $80-90 \%$ confluence. To detect the interaction between miR-497 and GLUT1 3'UTR, the cells were cotransfected with $50 \mathrm{nM}$ of either scramble or miR-497 mimics and $40 \mathrm{ng}$ of either pmirGLO-GLUT1-3'UTR-wild-type (WT) or pmirGLO-GLUT1-3'UTR-mutant (MUT) using Lipofectamine 2000 according to the manufacturer's protocols. The cells were collected $48 \mathrm{~h}$ after transfection and analyzed using the Dual-Luciferase ${ }^{\circledR}$ Reporter Assay system (Promega Corporation, Madison, WI, USA). A plasmid constitutively expressing Renilla luciferase was cotransfected as an internal control to correct for differences in transfection and harvesting efficiencies. The transfections were performed in duplicate, and at least three independent experiments were performed.

Western blotting. Protein was harvested with radioimmunoprecipitation assay buffer (Sigma-Aldrich, St. Louis, MO, USA), quantified and then resolved on a $1 \%$ sodium dodecyl sulfate (SDS)-polyacrylamide gel electrophoresis gel. The protein was then transferred onto nitrocellulose membrane, which was blocked in 5\% non-fat milk and incubated with the following primary antibodies: Rabbit monoclonal anti- $\beta$-actin (1:5,000; catalog no. 4970), rabbit monoclonal anti-GLUT1 (1:1,000; catalog no. 12939) (Cell Signaling Technology, Inc., Danvers, MA, USA). After being washed, the membranes were incubated with hydrogen peroxide and alkaline phosphatase-conjugated secondary antibodies (Abcam, Cambridge, UK). The proteins were detected by chemiluminescence using Bio-Rad Gel Imaging system (ChemiDoc MP System;

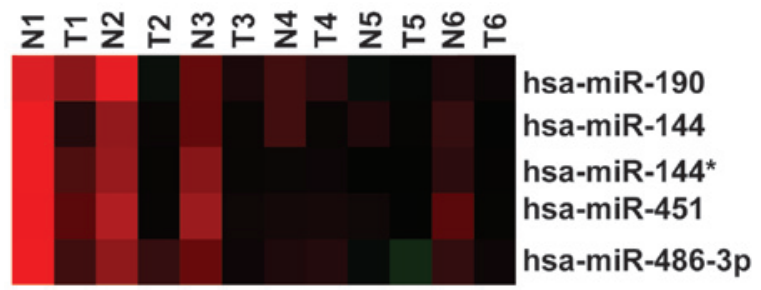

Figure 1. miR-144 expression is decreased in lung cancer. Selected heatmap of microRNA expression assay in lung cancer tissues compared with normal tissue, showing the decrease in miR-144 expression in the lung cancer tissues. $\mathrm{N}$, normal tissue; T, tumor; miR, microRNA; hsa, Homo sapiens.

Bio-Rad Laboratories, Hercules, CA, USA) and Pierce ${ }^{\mathrm{TM}}$ ECL Western Blotting Substrate (Thermo Fisher Scientific, Inc.).

Cell viability assays. A total of 5,000 cells/well were seeded into a 96-well plate and transfected with miR-144 mimics or miR-144 inhibitor (final concentration, $50 \mathrm{~nm}$ ). Following incubation for $48 \mathrm{~h}$ at $37^{\circ} \mathrm{C}$, the cells were labeled with Cell Counting kit-8 (CCK8) (Dojindo, Tabaru, Japan) for $1 \mathrm{~h}$. Cell viability was measured at an absorbance of $450 \mathrm{~nm}$ using Synergy ${ }^{\mathrm{TM}}$ H4 Hybrid Multi-mode Microplate Reader (BioTek Instruments, Inc., Winooski, VT, USA).

Measurement of extracellular lactate. In total, $5 \times 10^{5}$ cells were seeded into 60-nm dishes and transfected with miR-144 mimics or miR-144 inhibitor, then incubated in DMEM with $10 \%$ FBS overnight. Next, the media was removed, and the cells were incubated in DMEM without FBS. Subsequent tot incubation for $1 \mathrm{~h}$, the supernatant was collected. The concentration of lactate in the supernatant was measured using a colorimetric assay according to the manufacturer's protocols (Lactate Assay kit; BioVision Inc., Milpitas, CA, USA) .

RNA extraction and reverse transcription quantitative polymerase chain reaction ( $R T-q P C R)$. Total RNA was extracted from A549 cells using TRIzol Reagent (Invitrogen; Thermo Fisher Scientific, Inc.) in accordance with the manufacturer's protocol. miR expression was measured using a TaqMan MicroRNA Assay (Invitrogen; Thermo Fisher Scientific, Inc.), according to the manufacturer's protocol and treated with DNase ( $1 \mu \mathrm{g}$ RNA per 5 U DNase) (Invitrogen; Thermo Fisher Scientific, Inc.). cDNA was synthesized from $200 \mathrm{ng}$ of total RNA using the Universal cDNA Synthesis kit (Exiqon, Vedbaek, Denmark) in a $15 \mu$ l reaction. miRNA expression was measured using a TaqMan ${ }^{\circledR}$ MicroRNA Assay (Invitrogen; Thermo Fisher Scientific, Inc.), according to the manufacturer's protocol. cDNAs were then amplified using qPCR with TaqMan miRNA Assays Human Panel sequence-specific primers. PCR was performed in an Applied Biosystems 7500 Fast Real-Time PCR System (Invitrogen; Thermo Fisher Scientific, Inc.) under the following conditions: $95^{\circ} \mathrm{C}$ for $60 \mathrm{sec}$, followed by 40 cycles of $95^{\circ} \mathrm{C}$ for $5 \mathrm{sec}$ and $60^{\circ} \mathrm{C}$ for $40 \mathrm{sec}$. All samples were normalized to the internal control and fold changes were calculated using the $2^{-\Delta \Delta C q}$ quantification method (16). The expression of RNU48 served as an internal control. The primers used for miR-144 and RNU48 (Sigma-Aldrich) were as follows: miR-144, forward 5'-TAC AGTATAGATGAT-3' and reverse 5'-GTGCAGGGTCCG 
A

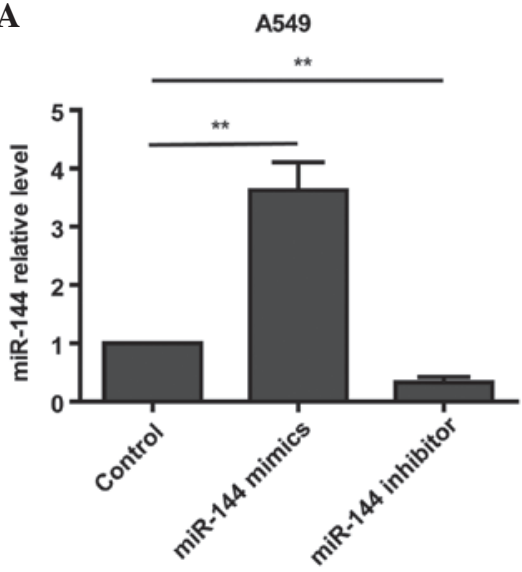

B

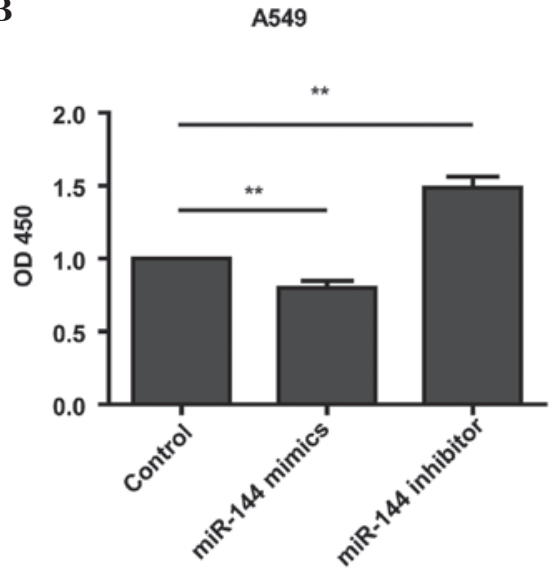

Figure 2. miR-144 inhibits the proliferation of lung cancer cells. (A) Reverse transcription-quantitative polymerase chain reaction analysis of miR-144 level in A549 cancer cells transfected with miR-144 mimics or inhibitor. (B) Cell Counting kit-8 assay results showing that the growth rate of miR-144-overexpressing A549 cancer cells was decreased compared with that of the control cells. Conversely, knockdown of miR-144 in the A549 cancer cells enhanced cell growth. ${ }^{* *} \mathrm{P}<0.01$. miR, microRNA; OD, optical density.

AGGT-3'; RNU48 forward 5'-TGATGATGACCCCAGGTA ACTC-3' and reverse 5'-GAGCGCTGCGGTGATG-3' (2,17).

Measurement of glucose uptake. Following $12 \mathrm{~h}$ of serum starvation, the cells were cultured in DMEM containing $25 \mathrm{mM}$ glucose. To measure the rate of glucose uptake, the cells were washed three times with phosphate-buffered saline (PBS) and then incubated for $3 \mathrm{~h}$ in DMEM containing $1 \mathrm{mCi} / \mathrm{ml}$ 2-deoxy-D-[1,2-3H]-glucose (PerkinElmer Inc., Waltham, MA, USA). The cells were washed three times with ice-cold PBS and solubilized in $1 \%$ SDS. A scintillation counter (Beckman LS 6000SC Scintillation Counter; Beckman Coulter, Brea, CA, USA) was used to determined the radioactivity of each aliquot. Each assay was performed in triplicate.

Statistical analysis. Statistical analysis was performed with SPSS version 17 software (SPSS Inc., Chicago, IL, USA). Analysis of variance were used to determined statistical significance. $\mathrm{P}<0.05$ was used to indicate a statistically significant difference.

\section{Results}

miR-144 expression is decreased in lung cancer tissue. First, a previously published dataset (ArrayExpress Accession no. E-MTAB-113; www.ebi.ac.uk/arrayexpress/) was downloaded, and the expression of miR-144 was analyzed in lung cancer tissue; the result showed that miR-144 was decreased in the lung cancer tissues compared with the normal lung tissues $(\mathrm{P}=0.0075)$ (Fig. 1). This result was consistent with that of the study by Zha et al (2).

miR-144 inhibits the proliferation of A549 cells. To assess the role of miR-144 in the growth of glioma cells, miR-144 mimic or miR-144 inhibitor was transfected into A549 cells at a final concentration of $50 \mathrm{nM}$. The RT-qPCR analysis revealed that miR-144 expression was markedly and specifically increased in the mimic-transfected cells and decreased in the inhibitor-transfected cells (Fig. 2A). A CCK8 assay showed that cell proliferation was decreased in the miR-144-overexpressing
A549 cells compared with the control cells (Fig. 2B). The study then investigated whether reducing miR-144 in the A549 cells enhanced cell proliferation. As expected, the loss of miR-144 function in the A549 cells treated with miR-144 inhibitor increased cell proliferation (Fig. 2B). Taken together, these results suggested that miR-144 plays a role as a tumor suppressor in A549 cell proliferation.

Decreased expression of miR-144 induces a metabolic shift in A549 cells. The present study next sought to investigate whether miR-144 was capable of inducing a metabolic shift in the A549 cells. Loss of miR-144 function in the A549 cells was found to increase glucose uptake and lactate secretion (Fig. 3A and B). Conversely, gain of miR-144 function in A549 cells treated with mimics reduced glucose uptake and lactate secretion (Fig. 3A and B).

To detect whether the miR-144-induced metabolic switch is required for miR-144-dependent cell proliferation, miR-144 inhibitor-transfected A549 cells were administered 2-deoxyglucose (2-DG), a glucose analog that inhibits glycolysis by its action on hexokinase, and analyzed cell proliferation with a CCK8 assay. The results demonstrated that inhibition of glycolysis induced by loss of miR-144 function is sufficient to block cell proliferation (Fig. 3C). Taken together, these results suggested that miR-144-induced glycolysis is responsible for the enhanced cell proliferation in miR-144-knockdown cells.

miR-144 suppresses the expression of GLUT1 by targeting its 3'-UTR. Two different mRNA target-predicting algorithms (TargetScan and miRanda $(18,19)$ were used to identify potential direct targets of miR-144 based on binding sites in the 3'UTR. Candidate genes that are involved in glycolysis were selected. mRNA target-predicting algorithms identified an area within the 3'-UTR of GLUT1 as a potential target of miR-144 (Fig. 4A). Western blot analysis confirmed that the overexpression of miR-144 decreased the protein level of GLUT1 (Fig. 4B), while knockdown of miR-144 increased the protein level of GLUT1 (Fig. 4B). To further determine whether GLUT1 is a direct target of miR-144, a luciferase assay was performed. The results showed that miR-144 effectively 
A

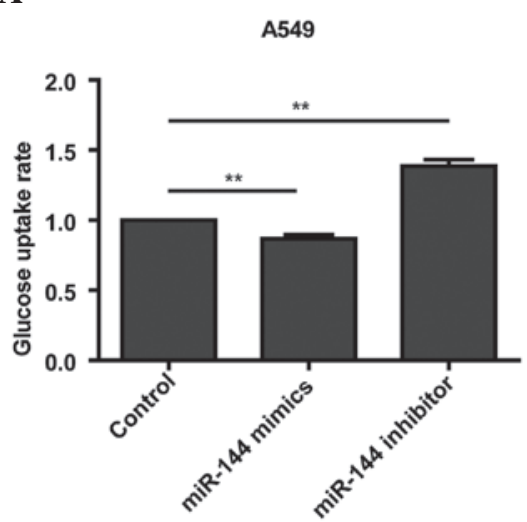

B

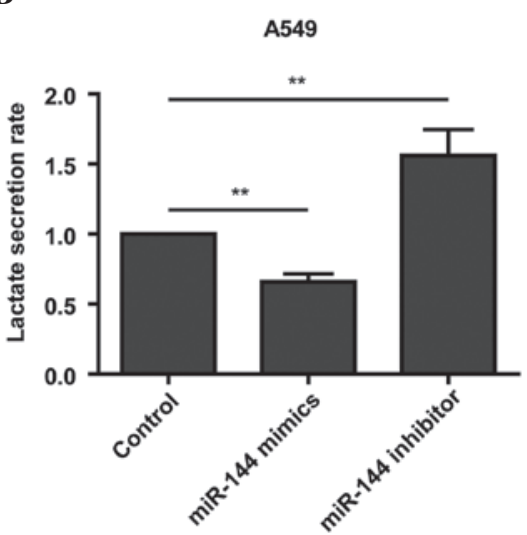

C
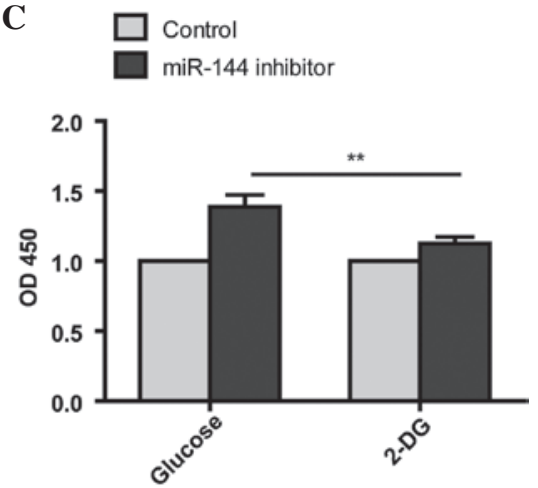

Figure 3. miR-144 induces a metabolic shift in lung cancer cells. (A) Glucose uptake assays showed that miR-144 decreases the rate of glucose uptake. (B) Lactate production assays showed that miR-144 blocks lactate secretion. (C) Cell Counting kit-8 assays showed that inhibition of glycolysis with 2-deoxy-D-glucose blocks the enhanced proliferate rate induced by the knockdown of miR-144. ${ }^{* *} \mathrm{P}<0.01$. miR, microRNA; OD, optical density.

A

Glut1 5'- aaUGUGAAGUAAAUACUGUa -3' miR-144 3'- ucAUGUAGUAGAUAUGACAu -5 '
B

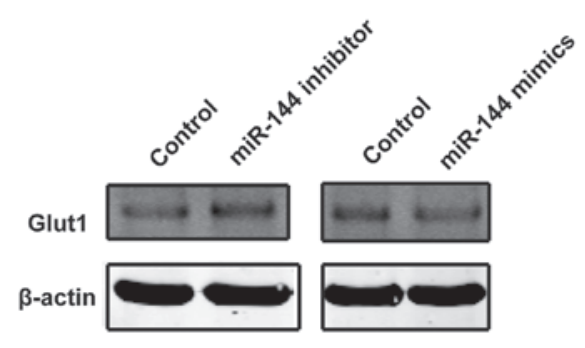

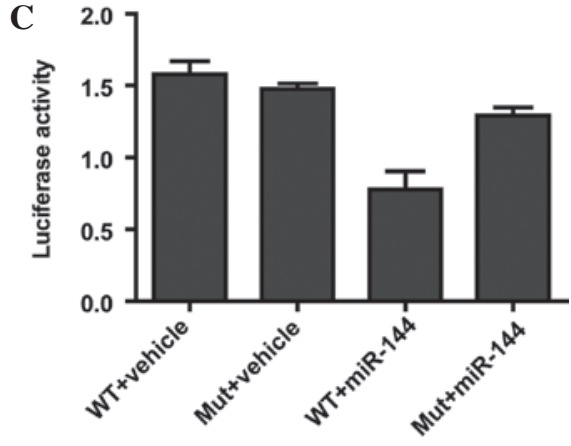

Figure 4. miR-144 suppresses the expression of GLUT1. (A) Sequences of miR-144 and the potential binding sites at the 3'UTR of GLUT1. (B) Western blot assays showed that the overexpression of miR-144 suppresses the expression of GLUT1, and the knockdown of miR-144 increases the expression of GLUT1. (C) Dual-luciferase assays showed that miR-144 suppresses the luciferase activity in the 3'UTR WT group, but not in the 3'UTR MUT group. WT, wild-type; MUT, mutant; miR, microRNA; UTR, untranslated region; GLUT1, glucose transporter 1.

suppressed the luciferase reporter activity of the WT vector, but that this decreased luciferase activity was reversed when cells were transfected with the MUT vector (Fig. 4C). Taken together, these results demonstrated that miR-144 directly regulates GLUT1 expression via binding sites in the 3'-UTR.

\section{Discussion}

miRNAs have attracted an increasing level of attention due to their key role in a number of biological events, including cell proliferation, cell motility, differentiation, metabolic switch and apoptosis (7). In recent years, the dysregulation of miR-144 has been associated with several types of human cancer. Significant miR-144 downregulation has been reported in osteosarcoma and mesothelioma $(11,12)$. miR-144 acts as a tumor suppressor in uveal melanoma by inhibiting cell proliferation and migration (20). Chen et al reported that downregulation of miR-144 in lung cancer inhibits proliferation and induces apoptosis and autophagy by targeting TP53-inducible glycolysis and apoptosis regulator (21). However, the function of miR-144 in lung tumorigenesis remains unclear.

In the present study, it was found that miR-144 expression was decreased in lung cancer compared with adjacent normal tissues. Previous studies have demonstrated that miR-144 acts as a tumor suppressor to inhibit cell proliferation in osteosarcoma and mesothelioma $(11,12)$. The results of the CCK8 assay in the present study also indicated that miR-144 decreased cell proliferation.

The molecular mechanisms responsible for shifting energy metabolism to the advantage of cancer cells are complicated and only partially understood (22). In the present study, it was found that miR-144 plays a significant role in regulating the metabolism of lung cancer cells. Knockdown of miR-144 increased glucose uptake rate and lactate secretion, while overexpression of miR-144 decreased glucose uptake and lactate secretion. The altered metabolism induced by miR-144 was required for lung cancer cell proliferation. Moreover, it was found that GLUT1 is a direct target of miR-144. The induction of GLUT1 enhanced aerobic glycolysis in the cancer cells. Dysregulation of miR-144 in lung cancer cells requires extensive investigation concerning its role in cancer cell metabolism.

In summary, the present study provides direct evidence that the decrease of miR-144 in lung cancer cells promotes cell glycolysis by increasing GLUT1 expression, thus enhancing cell growth. These results identify miR-144 as a molecular switch involved in the orchestration of the Warburg effect in lung cancer cells and as a potential therapeutic target for lung cancer. 


\section{References}

1. Mehan MR, Ayers D, Thirstrup D, Xiong W, Ostroff RM, Brody EN, Walker JJ, Gold L, Jarvis TC, Janjic N, et al: Protein signature of lung cancer tissues. PLoS One 7: e35157, 2012.

2. Zha W, Cao L, Shen Y and Huang M: Roles of Mir-144-ZFX pathway in growth regulation of non-small-cell lung cancer. PloS One 8: e74175, 2013.

3. Kroemer G and Pouyssegur J: Tumor cell metabolism: Cancer's Achilles' heel. Cancer Cell 13: 472-482, 2008.

4. Muñoz-Pinedo C, El Mjiyad N and Ricci JE: Cancer metabolism: Current perspectives and future directions. Cell Death Dis 3: e248, 2012.

5. Vander Heiden MG, Cantley LC and Thompson CB: Understanding the Warburg effect: the metabolic requirements of cell proliferation. Science 324: 1029-1033, 2009.

6. Bartel DP: MicroRNAs: Target recognition and regulatory functions. Cell 136: 215-233, 2009.

7. Jansson MD and Lund AH: MicroRNA and cancer. Mol Oncol 6: 590-610, 2012

8. Rottiers V and Näär AM: MicroRNAs in metabolism and metabolic disorders. Nat Rev Mol Cell Biol 13: 239-250, 2012.

9. Rayner KJ, Suárez Y, Dávalos A, Parathath S, Fitzgerald ML, Tamehiro N, Fisher EA, Moore KJ and Fernández-Hernando C: MiR-33 contributes to the regulation of cholesterol homeostasis Science 328: 1570-1573, 2010.

10. Wilfred BR, Wang WX and Nelson PT: Energizing miRNA research: A review of the role of miRNAs in lipid metabolism, with a prediction that miR-103/107 regulates human metabolic pathways. Mol Genet Metab 91: 209-217, 2007.

11. Namløs HM, Meza-Zepeda LA, Barøy T, Østensen IH, Kresse SH, Kuijjer ML, Serra M, Bürger H, Cleton-Jansen AM and Myklebost O: Modulation of the osteosarcoma expression phenotype by microRNAs. PloS One 7: e48086, 2012.

12. Guled M, Lahti L, Lindholm PM, Salmenkivi K, Bagwan I, Nicholson AG and Knuutila S: CDKN2A, NF2 and JUN are dysregulated among other genes by miRNAs in malignant mesothelioma-A miRNA microarray analysis. Genes Chromosomes Cancer 48: 615-623, 2009.
13. Akiyoshi S, Fukagawa T, Ueo H, Ishibashi M, Takahashi Y, Fabbri M, Sasako M, Maehara Y, Mimori K and Mori M: Clinical significance of miR-144-ZFX axis in disseminated tumour cells in bone marrow in gastric cancer cases. Br J Cancer 107: 1345-1353, 2012

14. Kalimutho M, Del Vecchio Blanco G, Di Cecilia S, Sileri P, Cretella M, Pallone F, Federici G and Bernardini S: Differential expression of miR-144* as a novel fecal-based diagnostic marker for colorectal cancer. J Gastroenterol 46: 1391-1402, 2011.

15. Zhang LY, Ho-Fun Lee V, Wong AM, Kwong DL, Zhu YH, Dong SS, Kong KL, Chen J, Tsao SW, Guan XY and Fu L: MicroRNA-144 promotes cell proliferation, migration and invasion in nasopharyngeal carcinoma through repression of PTEN. Carcinogenesis 34: 454-463, 2013.

16. Pfaffl MW: A new mathematical model for relative quantification in real-time RT-PCR. Nucleic Acids Res 29: e45, 2001.

17. Chen C, Ridzon DA, Broomer AJ, Zhou Z, Lee DH, Nguyen JT, Barbisin M, Xu NL, Mahuvakar VR, Andersen MR, et al: Real-time quantification of microRNAs by stem-loop RT-PCR. Nucleic Acids Res 33: e179, 2005.

18. Lewis BP, Burge CB and Bartel DP: Conserved seed pairing, often flanked by adenosines, indicates that thousands of human genes are microRNA targets. Cell 120: 15-20, 2005.

19. Betel D, Wilson M, Gabow A, Marks DS and Sander C: The microRNA.org resource: Targets and expression. Nucleic Acids Res 36(Database issue): D149-D153, 2008.

20. Sun L, Bian G, Meng Z, Dang G, Shi D and Mi S: MiR-144 inhibits uveal melanoma cell proliferation and invasion by regulating c-Met expression. PLoS One 10: e0124428, 2015.

21. Chen S, Li P, Li J, Wang Y, Du Y, Chen X, Zang W, Wang H, Chu H, Zhao G and Zhang G: MiR-144 inhibits proliferation and induces apoptosis and autophagy in lung cancer cells by targeting TIGAR. Cell Physiol Biochem 35: 997-1007, 2015.

22. Zhao Y, Butler EB and Tan M: Targeting cellular metabolism to improve cancer therapeutics. Cell Death Dis 4: e532, 2013. 